BULLETIN OF THE

AMERICAN MATHEMATICAL SOCIETY

Volume 79, Number 5, September 1973

\title{
FUNDAMENTAL GROUPS, NILMANIFOLDS AND ITERATED INTEGRALS ${ }^{1}$
}

\author{
BY KUO-TSAI CHEN
}

Communicated by S. S. Chern, January 20, 1973

Let $X$ be a connected $C^{\infty}$ manifold. Denote by $P(X)$ the total space of piecewise smooth paths in $X$. Choose a base point $x_{0}$. Denote by $P\left(X ; x_{0}\right)$ (resp. $\left.\Omega X\right)$ the space of piecewise smooth paths (resp. loops) from the base point $x_{0}$.

Let $k$ be the field of real (or complex) numbers. All differential forms are $k$-valued. Let $w_{1}, w_{2}, \ldots$ denote 1 -forms on $X$. For a piecewise smooth path $\alpha: I \rightarrow X$, let $f_{i}(t)=w_{i}(\alpha(t), \dot{\alpha}(t))$ be the value of the 1 -form $w_{i}$ at the tangent vector $\dot{\alpha}(t)$ of $X$. Define the $r$-time iterated integral $\int w_{1} \cdots w_{r}$ to be the $k$-valued function on $P(X)$ whose value at $\alpha$ is given by

$$
\left\langle\int w_{1} \cdots w_{r}, \alpha\right\rangle=\int_{0}^{1} \int_{0}^{t_{r}} \cdots \int_{0}^{t_{2}} f_{1}\left(t_{1}\right) d t_{1} \cdots f_{r-1}\left(t_{r-1}\right) d t_{r-1} f_{r}\left(t_{r}\right) d t_{r}
$$

when $r>0$ and $=1$ when $r=0$. At times, we shall also take $\int w_{1} \cdots w_{r}$ as its restriction on $\Omega X$ or $P\left(X ; x_{0}\right)$.

Let $F$ be the function algebra on $P(X)$ consisting of those functions whose value at each path $\alpha$ remains invariant under any piecewise smooth homotopy of $\alpha$ relative to $\dot{I}$. In this note, we shall consider the subspace of $F$ whose elements are linear combinations of iterated integrals. A characterization of this subspace in terms of the fundamental group $\pi_{1}(X)$ will be given.

We begin with a differential graded subalgebra $A$ of the exterior algebra $\Lambda(X)$. The following assumptions are made:

I. $d A^{0}=A^{1} \cap d \Lambda^{0}(X)$.

II. $\operatorname{dim} H^{1}(A)<\infty$.

III. The canonical homomorphism $H^{q}(A) \rightarrow H^{q}(X ; k)$ is an isomorphism when $q=1$ and is a monomorphism when $q=2$.

A primary example is the case of $A=\Lambda(X)$.

For $s \geqq 0$, denote by $F_{A}(s)$ the subspace of $F$ whose elements are linear combinations of iterated integrals of the type

AMS (MOS) subject classifications (1970). Primary 58A99; Secondary 49F05, 53C65.

Key words and phrases. Iterated integrals, path spaces, fundamental groups, differential forms, nilpotent Lie groups, nilmanifolds.

${ }^{1}$ Work supported in part by the National Science Foundation under NSF-GP-34257. 


$$
\int w_{1} \cdots w_{r}, \quad 0 \leqq r \leqq s, \quad w_{1}, \ldots, w_{r} \in A^{1} .
$$

Then $k=F_{A}(0) \subset \cdots \subset F_{A}(s) \subset \cdots$. Moreover $F_{A}=\bigcup F_{A}(s)$ turns out to be closed under multiplication. Let $F_{A}^{\prime}(s)\left(\operatorname{resp} . F_{A}^{\prime \prime}(s)\right)$ be obtained from $F_{A}(s)$ by restricting to $\Omega X$ (resp. $P\left(X ; x_{0}\right)$ ). Then $F_{A}^{\prime}=\bigcup F_{A}^{\prime}(s)$ and $F_{A}^{\prime \prime}=\bigcup F_{A}^{\prime \prime}(s)$ are algebras obtained from the algebra $F_{A}$ by restrictions.

Let $k \pi_{1}(X)$ be the group algebra of $\pi_{1}(X)$ over $k$, and let $N$ be the augmentation ideal, which is generated by all $\langle\alpha\rangle-1$, where $\langle\alpha\rangle$ denotes the homotopy class of a piecewise smooth loop at $x_{0}$. There is a pairing $F_{A}^{\prime} \times k \pi_{1}(X) \rightarrow k$ given by $(u,\langle\alpha\rangle) \mapsto\langle u, \alpha\rangle$ which is the value of the linear combination $u$ of iterated integrals at the loop $\alpha$.

THEOREM 1. With respect to the above pairing $F_{A}^{\prime}(s)=\left(N^{s+1}\right)^{\perp}, s \geqq 0$.

In order to outline a proof of this theorem, we recall that iterated integrals can be defined for forms of higher degrees in $A$. In relation to $\Omega X$, such iterated integrals form a differential graded algebra $A^{\prime}$ with an ascending filtration $\left\{A^{\prime}(s)\right\}$ (see [3]). Choose a suitable cubical chain complex $C_{*}(\Omega X)$ so that it has a descending filtration by the powers of its augmentation ideal. Let $\{B(s)\}$ be the dual ascending filtration for the cochain complex $C^{*}(\Omega X ; k)$. Then $B=\bigcup B(s)$ is a filtered subcomplex of $C^{*}(\Omega X ; k)$. Theorem 1 follows from comparing the spectral sequences of the filtered cochain complexes $A^{\prime}$ and $B$ and the fact that $F_{A}^{\prime} \approx H^{0}\left(A^{\prime}\right)$. Since the restriction map $F_{A}^{\prime \prime} \rightarrow F_{A}^{\prime}$ is surjective, we are also led to the next conclusion.

THEOREM 2. If $A^{0}$ separates points of $X$ and if $\bigcap N^{s}=0$, then $F_{A}^{\prime \prime}$, taken as an algebra of functions on the universal covering space $\tilde{X}$ of $X$ separates points of $\tilde{X}$.

This result is related to a work of Parsin [5]. He considered the case where $X$ is a Riemann surface, and $A$ is the algebra of holomorphic differential forms. Our assumption III does not hold for his case.

If $\pi_{1}(X)$ is finitely generated torsion free nilpotent, we can show that $F_{A}^{\prime}$ is the coordinate ring of a simply connected nilpotent Lie group $G$ having a uniform discrete subgroup $\Gamma \approx \pi_{1}(X)$. By constructing an injection $F_{A}^{\prime} \rightarrow F_{A}^{\prime \prime}$, we obtain the next assertion.

THEOREM 3. If $X$ is a connected $C^{\infty}$ manifold with $\pi_{1}(X)$ being finitely generated torsion free nilpotent, then there exists a compact nilmanifold $M(X)$ and a $C^{\infty}$ map $X \rightarrow M(X)$ which induces an isomorphism for the fundamental groups.

Observe that $G$ can be taken as the Malcev completion of $\Gamma \approx \pi_{1}(X)$. 
The nilmanifold $M(X)=G / \Gamma$ associated to $X$ is essentially unique (see [1] or [6]).

REMARK. In the case of $X$ being Riemannian, there is a canonical injection $F_{A}^{\prime} \rightarrow F_{A}^{\prime \prime}$ with $A=\Lambda(X)$ so that, in the theorem, the map $X \rightarrow M(X)$ is canonical. If $X$ is, moreover, real analytic, so is the map.

The details of these theorems in a somewhat more general context will appear elsewhere.

\section{BIBLIOGRAPHY}

1. L. Auslander, L. Green and F. Hahn, Flows on homogeneous spaces, Ann. of Math. Studies, no. 53, Princeton Univ. Press, Princeton,, N.J., 1963. MR 29 \# 4841.

2. K. T. Chen, Algebras of iterated path integrals and fundamental groups, Trans. Amer. Math. Soc. 156 (1971), 359-379. MR 43 \# 1069.

3. - Iterated integrals of differential forms and loop space homology, Ann. of Math. 97 (1973), 217-246.

4. A. Mal'cev, On a class of homogeneous spaces, Izv. Akad. Nauk SSSR Ser. Mat. 13 (1949), 9-32; English transl., Amer. Math. Soc. Transl. (1) 9 (1962), 276-307. MR 10, 507.

5. A. N. Parsin, A generalization of Jacobian variety, Izv. Akad. Nauk SSSR Ser. Mat. 30 (1966), 175-182; English transl., Amer. Math. Soc. Transl. (2) 84 (1969), 187-196. MR 33 \#4956.

6. M. S. Raghunathan, Discrete subgroups of Lie groups, Springer-Verlag, Berlin and New York, 1972.

Department of Mathematics, University of Illinois, Urbana, Illinois 61801 